JGSP 10 (2007) 29-40

\title{
TUBE FORMULA, BEREZINIANS, AND DWORK FORMULA
}

\author{
HOVHANNES M. KHUDAVERDIAN
}

Communicated by Theodore Voronov

\begin{abstract}
We consider an example of tubes of hypersurfaces in Euclidean space and generalise the tube formula to supercase. By this we assign to a point of the hypersurface in superspace a rational characteristic function. Does this rational function appear when we calculate the $\zeta$-function of an arithmetic variety?
\end{abstract}

\section{Introduction}

I would like to make a remark on relations between tube formula and Dwork formula for $\zeta$-function for arithmetic varietes. I have been thinking about this relation and have discussed it for several years with many colleagues. In particular I spoke about it in Białowieża last summer. Recently a very interesting paper [1] appeared in the web which touches on a related circle of ideas.

\section{Tubes of Hypersurfaces}

Recall some simple facts concerning tubes of hypersurfaces in Euclidean space.

Let $M$ be a surface in Euclidean space $\mathbb{E}^{n+1}$. By a tube we shall understand the set of points in $\mathbb{E}^{n+1}$ that are at distance $h$ from $M, h \geq 0$. If $M$ is an orientable hypersurface (surface of codimension 1), then a direction of normal vector can be chosen. This defines sign of the distance between a point and the surface. In such a case the tube of radius $h$ is the disconnected union of two half-tubes $M_{h}$ and $M_{-h}$. We consider here only oriented hypersurfaces and later denote by $M_{h}$ a half-tube for any $h \in \mathbb{R}$. The $n$-dimensional volumes of tubes and half-tubes are polynomials in $h$ if $h$ is small enough. These formulae can be traced to Steiner (1840), who derived them for a polygon and a polyhedron. In 1939 Weyl gave general formulae for polynomials expressing volumes of tubes and half-tubes. The coefficients of these polynomials are integrals of expressions which are formed from the second quadratic form at $n$-dimensional surface. For 
tubes (not half-tubes) these coefficients do not change under isometries of the surface; they are expressed via internal curvature tensor. An excellent exposition on tube formula containing full references is given in [2].

Consider first a toy example. Let $M$ be the boundary of a convex polygon. Then it is evident that vol $M_{h}=\operatorname{vol} M+2 \pi h$ and $\operatorname{vol}\left(M_{h} \cup M_{-h}\right)=2 \mathrm{vol} M$. Henceforth the volume of a $k$-dimensional surface $M$ is denoted vol $M$. If $M$ is onedimensional, then vol $M$ is length, if $M$ is two-dimensional, then vol $M$ is area.

Now let $M$ be a closed orientable hypersurface in $\mathbb{E}^{n+1}$ and $\mathbf{n}$ be normal unit vector field of $M$. Consider new coordinates $\left(u^{1}, \ldots, u^{n}, t\right)$ in a tubular neighborhood of $M$ defined by the relations $x^{a}(u, t)=x^{a}(u)-t n^{a}(x(u))$, where $x^{a}=x^{a}(u)$ is a local parameterisation of $M$. Straightforward calculations show that the Jacobian of transformation from Cartesian coordinates $\left(x^{1}, \ldots, x^{n+1}\right)$ to these new coordinates

$$
\begin{aligned}
J & =\operatorname{det}\left(\frac{\partial\left(x^{1}, \ldots, x^{n+1}\right)}{\partial\left(u^{1}, \ldots, u^{n}, t\right)}\right)=\operatorname{det}\left(\frac{\partial x^{a}(u)}{\partial u^{i}}-t \frac{\partial n^{a}(x(u))}{\partial u^{i}}, n^{a}(x(u))\right) \\
& =\sqrt{\operatorname{det} g_{i j}(u)} \operatorname{det}(1+t S(u)) .
\end{aligned}
$$

Here $g_{i j}=\frac{\partial x^{a}}{\partial u^{i}} \frac{\partial x^{a}}{\partial u^{j}}$ is induced Riemannian metric on $M$ (the first quadratic form). It defines volume form $d \sigma_{u}=\sqrt{\operatorname{det} g_{i j}(u)} d^{n} u$ on the surface $M$ in the parameterisation $x^{a}(u)$. The linear operator $S$ is variant of second quadratic form. It is Weingarten (shape) operator defined by the relation $S \mathbf{v}=-\partial_{\mathbf{v}} \mathbf{n}$ for an arbitrary tangent vector $\mathbf{v}: S_{i}^{j} \frac{\partial x^{a}}{\partial u^{j}}=-\frac{\partial n^{a}}{\partial u^{i}}$ and $S_{i}^{j}=g^{j k} n^{a} \frac{\partial^{2} x^{a}}{\partial u^{k} \partial u^{i}}$. (Henceforth we will not distinguish between upper and lower indices in Euclidean space and we suppose summation over repeated indices.)

Using the Jacobian (1) one can easy calculate the volume of the half-tube $M_{h}$ for small $h$. Let $\rho(t)(t \in \mathbb{R})$ be an arbitrary (smooth) function such that it vanishes outside of sufficiently large neighborhood of zero. Consider the integral $\int \rho(t(x)) \mathrm{d}^{n+1} x, t\left(x^{a}\right)$ being the distance between the point $\left(x^{1}, \ldots, x^{n}\right)$ and surface $M$. On one hand this integral is equal to $\int \rho(t) \operatorname{vol} M_{t} \mathrm{~d} t$. On the other hand, by using formula (1) we arrive at

$$
\int \rho(t(x)) \mathrm{d}^{n+1} x=\int \mathrm{d} t \rho(t)\left(\int_{M} \operatorname{det}(1+t S(u)) \mathrm{d} \sigma_{u}\right) .
$$

In particular it follows that if $M$ is closed and $h$ is small enough then the volume 
of half-tube is equal to

$$
\operatorname{vol} M_{h}=\int_{M} \operatorname{det}(1+h S(u)) \mathrm{d} \sigma_{u}=\sum_{k=0}^{n} c_{k} h^{k} .
$$

Coefficients $c_{k}$ are as follows: $c_{0}=\int_{M} \mathrm{~d} \sigma=\operatorname{vol}(M), c_{1}=\int_{M} \operatorname{Tr} S \mathrm{~d} \sigma$, the integral of mean curvature over surface, etc. The last coefficient $c_{n}=\int_{M} \operatorname{det} S \mathrm{~d} \sigma$ is equal to the volume of unit $n$-dimensional sphere multiplied by the degree of Gaussian map $M \stackrel{\mathbf{n}(u)}{\longrightarrow} S^{n}$. (If $n$ is even then the degree is equal up to a factor to Euler characteristic $\chi(M))$. E.g. if $M$ is two-dimensional closed (oriented) hypersurface in $\mathbb{E}^{3}$, then $\operatorname{det}(1+t S)=1+t \operatorname{Tr}+t^{2} \operatorname{det} S=1+t H+t^{2} K$, where $H=k_{1}+k_{2}$ is mean curvature, $K=k_{1} k_{2}$ is Gaussian curvature $\left(k_{1,2}\right.$ are principal curvatures). The volume (area) of half-tube $M_{h}$ is equal to vol $M_{h}=$ $\operatorname{vol} M+h \int_{M} H \mathrm{~d} \sigma+h^{2} \int_{M} K \mathrm{~d} \sigma=\operatorname{vol} M+h \int_{M} H \mathrm{~d} \sigma+2 \pi \chi(M) h^{2}$. Respectively, the volume of the tube $M_{h} \cup M_{-h}$ is equal to $2 \operatorname{vol} M+4 \pi \chi(M) h^{2}$.

Summarizing we can say the following: To an arbitrary hypersurface $M$ in $\mathbb{E}^{n+1}$ one can assign a local characteristic polynomial

$$
P_{M}(t, x(u))=\operatorname{det}(1+t S(u))
$$

and its integral over the surface, the characteristic polynomial

$$
P_{M}(t)=\int_{M} P_{M}(t, u) \mathrm{d} \sigma_{u}=\int_{M} \operatorname{det}(1+t S(u)) \mathrm{d} \sigma_{u} .
$$

The local characteristic polynomial $P_{M}(t, u)$ defines a measure density in a vicinity of a point $x(t, u)$ in tubular neighborhood of oriented hypersurface $M$. If $f\left(x^{a}\right)$ is an arbitrary function which decreases rapidly enough and vanishes outside sufficiently large tubular neighborhood of the surface $M$, then

$$
\int f(x) \mathrm{d}^{n+1} x=\int \mathrm{d} t\left(\int f(x(u, t)) P_{M}\left(t, u^{i}\right) \mathrm{d} \sigma_{u}\right) .
$$

In particular, the polynomial $P_{M}(h)$ measures the volume of the half tube $M_{h}$ if $M$ is closed hypersurface.

\section{Dual Approach}

In the previous section we considered surfaces specified by parametric equations $x^{i}=x^{i}(u)$. It is very useful to develop a dual approach, i.e., to write the integrals 
for hypersurface defined by an equation $\Phi(x)=0$. Formulae written in this language becomes much more transparent and easier to generalise to supercase.

If we consider a reparameterisation invariant integral $\int A\left(x, \frac{\partial x}{\partial u}, \frac{\partial^{2} x}{\partial u \partial u} \ldots\right) \mathrm{d}^{n} u$ over a surface specified by parametric equations $x^{i}=x^{i}(u)$, then the integrand $A$ is a density, which obeys the condition

$$
A\left(x, \frac{\partial x}{\partial \tilde{u}}, \frac{\partial^{2} x}{\partial \tilde{u} \partial \tilde{u}} \ldots\right)=A\left(x, \frac{\partial x}{\partial u}, \frac{\partial^{2} x}{\partial u \partial u} \ldots\right) \cdot \operatorname{det}\left(\frac{\partial u}{\partial \tilde{u}}\right)
$$

if we consider new parameterisation $x(\tilde{u})=x(u(\tilde{u}))$. In the dual approach we come to the integral $\int A\left(\frac{\partial \Phi(x)}{\partial x}, \frac{\partial^{2} \Phi(x)}{\partial x \partial x}, \ldots\right) \delta(\Phi) \mathrm{d}^{n+1} x$, if a surface is defined by an equation $\Phi(x)=0$. The function $A$ in this integral is a dual density. It obeys the condition

$$
\left.A\left(\frac{\partial \tilde{\Phi}(x)}{\partial x}, \frac{\partial^{2} \tilde{\Phi}(x)}{\partial x \partial x}, \ldots\right)\right|_{\Phi=0}=\left.G(x) A\left(\frac{\partial \Phi(x)}{\partial x}, \frac{\partial^{2} \Phi(x)}{\partial x \partial x}, \ldots\right)\right|_{\Phi=0}
$$

if $\tilde{\Phi}(x)=G(x) \Phi(x)$. This condition guarantees that the integral $\int A \delta(\Phi) \mathrm{d}^{n+1} x$ does not change if we replace a function $\Phi$ defining the surface $M$ by the new function $\tilde{\Phi}=G \Phi\left(\left.G\right|_{M} \neq 0\right)$.

Proposition 1. The function

$$
A_{\mathrm{vol}}(\partial \Phi)=\sqrt{\partial_{a} \Phi \partial_{a} \Phi}
$$

defines the dual density corresponding to the volume element at $M$. If the hypersurface $M$ is given by an equation $\Phi(x)=0$, then

$$
\operatorname{vol} M=\int A_{\mathrm{vol}}(\partial \Phi) \delta(\Phi) \mathrm{d}^{n+1} x .
$$

The function

$$
A_{\mathrm{mcurv}}\left(\partial \Phi, \partial^{2} \Phi\right)=-\partial_{a} \partial_{a} \Phi+\frac{\partial_{a} \Phi \partial_{b} \Phi \partial_{a} \partial_{b} \Phi}{\partial_{c} \Phi \partial_{c} \Phi}
$$

defines density corresponding to mean curvature. At any point $x$ of the surface $M$ defined by the equation $\Phi(x)=0$ the ratio $A_{\mathrm{mcurv}} / A_{\mathrm{vol}}$ of these densities is equal to the mean curvature $H(x)$

$$
\left.H\left(\partial \Phi, \partial^{2} \Phi\right)\right|_{x: \Phi(x)=0}=\left.\frac{A_{\mathrm{mcurv}}\left(\partial \Phi, \partial^{2} \Phi\right)}{A_{\mathrm{vol}}(\partial \Phi)}\right|_{x: \Phi(x)=0} .
$$


We have

$$
\int A_{\text {mcurv }}\left(\partial \Phi, \partial^{2} \Phi\right) \delta(\Phi) d^{n+1} x=\underbrace{\int H d \sigma_{u}=\int n^{a} g^{k r} x_{k r}^{a} \sqrt{\operatorname{det}\left(g_{i j}\right)} d^{n} u}_{\text {integral of the mean curvature over } M} .
$$

Proof: If we replace $\Phi \rightarrow G(x) \Phi(x)$, then $\left.\left.\partial_{a} \Phi\right|_{\Phi=0} \rightarrow G \partial_{a} \Phi\right|_{\Phi=0}$ and $\left.\left.\partial_{a} \partial_{b} \Phi\right|_{\Phi=0} \rightarrow G \partial_{a} \partial_{b} \Phi\right|_{\Phi=0}+\left.\partial_{a} G \partial_{b} \Phi\right|_{\Phi=0}+\left.\partial_{b} G \partial_{a} \Phi\right|_{\Phi=0}$. This implies that $A_{\text {mcurv }}$ and $A_{\text {vol }}$ obey condition (7) and are dual densities. To prove that the density $A_{\mathrm{vol}}$ defines volume, note that if the hypersurface is given by the equation $\Phi(x)=x^{n+1}-f\left(x^{1}, \ldots, x^{n}\right)$, then we have

$$
\int A_{\mathrm{vol}}(\Phi) \delta(\Phi) \mathrm{d}^{n+1} x=\int \sqrt{1+f_{1}^{2}+\cdots+f_{n}^{2}} \mathrm{~d} x^{1} \mathrm{~d} x^{2} \ldots \mathrm{d} x^{n} .
$$

Now consider the ratio $A_{\text {mcurv }} / A_{\text {vol }}$. If $\Phi \rightarrow G \Phi$, then $A_{\text {mcurv }} /\left.A_{\text {vol }}\right|_{\Phi=0}$ remains unchanged. Hence it is a well-defined function on the surface $M$. For any point on $M$ one can consider adjusted Cartesian coordinates in the ambient Euclidean space such that $\Phi(x)=x^{n+1}-A_{i j} x^{i} x^{j}+o\left(x^{2}\right)(i, j=1, \ldots, n)$ in the vicinity of this point. Mean curvature at this point is equal to $H=A_{i i}$. The RHS of formula (10) gives the same answer.

Remark 2. Note that according to the general philosophy one can come to the dual density corresponding to mean curvature by taking the variational derivative of the volume functional

$$
\begin{aligned}
\frac{\delta}{\delta \Phi}\left(\int \sqrt{\partial_{a} \Phi \partial_{a} \Phi} \delta(\Phi) d^{n+1} x\right) & =-\frac{\partial_{a} \Phi \partial_{a} \Phi}{\sqrt{\partial_{c} \Phi \partial_{c} \Phi}}+\frac{\partial_{a} \Phi \partial_{b} \Phi \partial_{b} \partial_{a} \Phi}{\left(\partial_{c} \Phi \partial_{c} \Phi\right)^{3 / 2}} \\
& =\frac{A_{\mathrm{mcurv}}\left(\partial \Phi, \partial^{2} \Phi\right)}{A_{\mathrm{vol}}(\partial \Phi)}
\end{aligned}
$$

Now we shall find an expression for characteristic polynomial (4) in the dual approach.

Consider the following expression:

$M_{a b}\left(\partial \Phi, \partial^{2} \Phi\right)=-\partial_{a} \partial_{b} \Phi-\frac{\partial_{a} \Phi \partial_{b} \Phi \partial_{d} \partial_{d} \Phi}{\partial_{c} \Phi \partial_{c} \Phi}+\frac{\partial_{a} \Phi \partial_{d} \Phi \partial_{d} \partial_{b} \Phi+\partial_{b} \Phi \partial_{d} \Phi \partial_{d} \partial_{a} \Phi}{\partial_{c} \Phi \partial_{c} \Phi}$.

Recall that we do not distinguish between upper and lower indices and implicitly understand summation over repeated indices. 
Proposition 3. Formula (11) defines a matrix-valued dual density. The ratio of this dual density and the dual density $A_{\mathrm{vol}}(\partial \Phi)=\sqrt{\partial_{a} \Phi \partial_{a} \Phi}$ defines a linear operator $\mathcal{S}$ on $\mathbb{E}^{n+1}$ depending on a point of the surface $M$ specified by an equation $\Phi(x)=0$ :

$$
\left.\mathcal{S}_{a b}\left(\partial \Phi, \partial^{2} \Phi\right)\right|_{x: \Phi(x)=0}=\left.\frac{M_{a b}\left(\partial \Phi, \partial^{2} \Phi\right)}{\sqrt{\partial_{c} \Phi \partial_{c} \Phi}}\right|_{x: \Phi(x)=0} .
$$

The linear operator $\mathcal{S}$ is the direct sum of the Weingarten (shape) operator $S$ acting on vectors tangent to $M$ and the scalar operator of the multiplication by the mean curvature on vectors orthogonal to $M$ :

$$
\mathcal{S}=S \oplus H, \text { if } \mathbf{v}=\mathbf{v}_{\text {tangent }}+\mathbf{v}_{\text {orthogonal }}, \mathcal{S} \mathbf{v}=S \mathbf{v}_{\text {tangent }}+H \mathbf{v}_{\text {orthogonal }}
$$

where $H=\operatorname{Tr} S$ is mean curvature at point $x \in M$.

The relation

$$
\operatorname{det}(1+t \mathcal{S}(x))=\operatorname{det}(1+t S(x))(1+t H(x))
$$

holds for an arbitrary point of the surface $M$, and the local characteristic polynomial of the surface $M$ is given by the formula

$$
P_{M}(x, t)=\frac{\operatorname{det}(1+t \mathcal{S}(x))}{(1+t H(x))} .
$$

Proof: In the same way as above, one can see that formula (11) defines a matrixvalued dual density. Hence $\mathcal{S}_{a b}$ is well-defined at the points $x: \Phi(x)=0$ as the ratio of two densities. It is easy to see that for an arbitrary point $x$ on $M$ in adjusted Cartesian coordinates $\mathcal{S}_{i j}=A_{i j}, \mathcal{S}_{0 i}=\mathcal{S}_{i 0}=0$ and $\mathcal{S}_{n+1, n+1}=A_{i i}=H$ $(i, j=1, \ldots, n)$. This implies (13) and (14).

The dual analog of the formula (6) has the following appearance: $\int f(x) \mathrm{d}^{n+1} x=$

$$
\int \mathrm{d} t\left(\int f\left(x^{a}-t n^{a}(\partial \Phi)\right) \frac{\operatorname{det}\left(1+t \mathcal{S}\left(\partial \Phi, \partial^{2} \Phi\right)\right)}{A_{\text {vol }}(\partial \Phi)+t A_{\text {mcurv }}\left(\partial \Phi, \partial^{2} \Phi\right)} A_{v o l}^{2}(\partial \Phi) \delta(\Phi) \mathrm{d}^{n+1} x\right)
$$

where $n^{a}(\partial \Phi(x))=\frac{\partial_{a} \Phi(x)}{A_{\text {vol }}}=\frac{\partial_{a} \Phi(x)}{\sqrt{\partial_{b} \Phi(x) \partial_{b} \Phi(x)}}$ is a unit normal vector field to the surface $\Phi=0$ at the point $x: \Phi(x)=0$. (Note that the surface defined by an equation $\Phi=0$ is orientable.) 


\section{Tube Formula for Hypersurfaces in Superspace}

Now we analyze how our constructions look in a superspace. We will see that the local characteristic function of surfaces in a superspace appearing in the tube formula is no longer a polynomial. It is a rational function.

Consider an $(n+1 \mid 2 m)$-dimensional Euclidean superspace with coordinates $z^{A}=$ $\left(x^{a}, \theta^{\alpha}\right)(a=1, \ldots, n+1, \alpha=1, \ldots, 2 m)$, where $x^{a}$ are even coordinates and $\theta^{\alpha}$ are odd ones $\left(x^{a} x^{b}=x^{b} x^{a}, x^{a} \theta^{\beta}=\theta^{\beta} x^{a}\right.$, but $\left.\theta^{\alpha} \theta^{\beta}=-\theta^{\beta} \theta^{\alpha}\right)$, with a Riemannian metric $G_{A B}$ such that $G_{A B} z^{A} z^{B}=x^{a} x^{a}+2 \theta^{1} \theta^{2}+\cdots+2 \theta^{2 m-1} \theta^{2 m}$. A hypersurface, i.e., a (1|0)-codimensional (or $(n-1 \mid 2 m)$-dimensional) surface can be specified by parametric equations: $z^{A}=z^{A}(w)$, where $w^{I}=\left(u^{i}, \eta^{\mu}\right)=$ $\left(u^{1}, \ldots, u^{n-1} ; \eta^{1}, \ldots \eta^{2 m}\right), u^{i}$ are even and $\eta^{\mu}$ are odd parameters. In the dual approach a hypersurface can be defined by an equation $\Phi(z)=0$, where $\Phi$ is an even function.

Two words about integration in superspace: $\int \theta \mathrm{d} \theta=1$ and $\int \theta^{\alpha} \mathrm{d} \theta^{\beta}=0$ if $\alpha \neq \beta$. Let $f(z)=f(x, \theta)=f_{0}(x)+f_{\alpha}(x) \theta^{\alpha}+\cdots+f_{1 \ldots q}(x) \theta^{1} \ldots \theta^{q}$ be a function on the $p \mid q$-dimensional superspace. Then

$$
\int f(z) \mathrm{d}^{p+q} z=\int f(x, \theta) \mathrm{d}^{p} x \mathrm{~d}^{q} \theta=\int f_{1 \ldots q}(x) \mathrm{d}^{p} x .
$$

The Jacobian of a coordinate transformation $z^{A}=z^{A}(\tilde{z}),\left(z^{A}=\left(x^{a}, \theta^{\alpha}\right)\right.$, is given by the Berezinian (superdeterminant) of the matrix

$$
\left(\frac{\partial z^{A}}{\partial \tilde{z}^{A^{\prime}}}\right)=\left(\begin{array}{cc}
\frac{\partial x^{a}(\tilde{x}, \tilde{\theta})}{\partial \tilde{x}^{a^{\prime}}} & \frac{\partial x^{a}(\tilde{x}, \tilde{\theta})}{\partial \tilde{\theta}^{\alpha^{\prime}}} \\
\frac{\partial \theta^{\alpha}(\tilde{x}, \tilde{\theta})}{\partial \tilde{x}^{a^{\prime}}} & \frac{\partial \theta^{\alpha}(\tilde{x}, \tilde{\theta})}{\partial \tilde{\theta}^{\alpha^{\prime}}}
\end{array}\right) .
$$

(We suppose that all functions of $x$ are smooth and rapidly decreasing at infinity). The Berezinian of an even $p|q \times p| q$ matrix $M$ is given by the following formula

$$
\operatorname{Ber} M=\operatorname{Ber}\left(\begin{array}{ll}
M_{00} & M_{01} \\
M_{10} & M_{11}
\end{array}\right)=\frac{\operatorname{det}\left(M_{00}-M_{01} M_{11}^{-1} M_{10}\right)}{\operatorname{det} M_{11}} .
$$

Here $M_{00}, M_{11}$ are $p \times p$ and $q \times q$ matrices with even entries and $M_{01}, M_{10}$ are $p \times q$ and $q \times p$ matrices with odd entries.

The formulae of the previous sections for the first quadratic form, mean curvature and Weingarten operator can be easily extended to the supercase. We just have to be cautious with the sign rule and consider Ber instead det. For example if a hypersurface is given by a parameterisation $z^{A}=z^{A}(w)$, then the first quadratic 
form is defined by the matrix: $g_{I J}=\frac{\partial z^{A}}{\partial w^{I}} G_{A B} \frac{\partial z^{B}}{\partial w^{J}}(-1)^{p(B)(p(J)+1)}$. (By $p(A)$ we denote the parity of the corresponding coordinate $z^{A}$.) The volume element is given by $\sqrt{\operatorname{Ber} g_{I J}}$ and volume is given by the integral $\int \sqrt{\operatorname{Ber} g_{I J}} \mathrm{~d}^{2 p+q} w$. For hypersurfaces the dual density corresponding to the volume form is equal to $A_{\mathrm{vol}}=\sqrt{\partial_{A} \Phi G^{A B} \partial_{B} \Phi(-1)^{p(B)}}$. Calculations in the dual approach for hypersurfaces are typically easier.

The tube formula for a hypersurface contains the local characteristic function

$$
R_{M}(t, w)=\operatorname{Ber}(1+t S(w)) .
$$

The essential difference with the previous case (see (4)) is that this local function is no longer a polynomial in $t$, because the Berezinian is a rational function of the matrix entries.

Recall the following important properties of the Berezinian of a linear operator (see [3]). Let $A$ be a linear operator in a $p \mid q$-dimensional space. Let $R_{A}(t)=$ $\operatorname{Ber}(1+t A)$ be its characteristic function. (We suppose that $A$ is an even operator.) Then

- $R_{A}(t)=\operatorname{Ber}(1+A t)$ is a rational function, the ratio of polynomials of degrees $p$ and $q$ respectively:

$$
R_{A}(t)=\operatorname{Ber}(1+A t)=\frac{1+a_{1} t+\cdots+a_{p} t^{p}}{1+b_{1} t+\cdots+b_{q} t^{q}} .
$$

- The expansion of the characteristic function at zero leads to traces of the exterior powers of the operator $A$ :

$$
\operatorname{Ber}(1+t A)=\sum_{k=0}^{\infty} c_{k}(A) t^{k}, \quad \text { where } \quad c_{k}(A)=\operatorname{Tr} \wedge^{k} A .
$$

- The expansion of the characteristic function at infinity leads to traces of the exterior powers of the inverse matrix:

$$
\operatorname{Ber}(1+t A)=\sum_{k=q-p}^{\infty} c_{-k}^{*}(A) t^{-k}, \quad c_{-k}^{*}(A)=\operatorname{Ber} A \cdot \operatorname{Tr} \wedge^{p-q+k} A^{-1} .
$$

- The sequences $c_{k}(k=0,1,2, \ldots)$ and $c_{k}^{*}(k=p-q, p-q-1, \ldots)$ are recurrent sequences with period $q$. Moreover, the sequence $\gamma_{k}=c_{k}-c_{k}^{*}$ $k \in \mathbb{Z}$ is a recurrent sequence with period $q$. 
- The following important formula holds: $\operatorname{Ber} A=\frac{\operatorname{Ber}^{+} A}{\operatorname{Ber}^{-} A}$, where $\operatorname{Ber}^{ \pm}$are invariant polynomial functions of the matrix entries of an operator $A$ (in fact, polynomials of $c_{k}=\operatorname{Tr} \wedge^{k} A$ ). If $A$ is presented by a diagonal matrix $\operatorname{diag}\left[\lambda_{1}, \ldots, \lambda_{p} ; \mu_{1}, \ldots, \mu_{q}\right]$, then

$$
\operatorname{Ber}^{+} A=R \cdot \prod_{a=1}^{p} \lambda_{a}, \quad \operatorname{Ber}^{-} A=R \cdot \prod_{\alpha=1}^{q} \mu_{a}
$$

where

$$
R=\prod_{a=1, \alpha=1}^{p, q}\left(\lambda_{a}-\mu_{\alpha}\right)
$$

is the resultant of numerator and denominator of the fraction $R_{K}(t)$.

Note that polynomials arising from the direct application of the original formula (16) are not invariant polynomials of matrix entries and they, in general, have degrees $p+p q$ and $q+p q$ respectively.

Applications of these results to RHS of the tube formula (17) gives information about the structure of differential-geometrical invariants of hypersurfaces in superspace.

Unlike the ordinary case where integration of a polynomial function (4) over the surface leads to a polynomial function (5), integration of the rational local characteristic function of a surface in a superspace leads in general to a non-rational function.

\section{Discussion}

Berezinians and characteristic functions of linear operators in superspace can naturally appear in situations which originally are not related to anything "super".

Consider the following example. Let $A$ be a linear operator in an ordinary linear space $V$. Suppose that a linear subspace $M$ of $V$ is invariant with respect to the action of the operator $A: A \mathbf{v} \in M$ for $\mathbf{v} \in M$. Thus the action of the operator $A$ is well-defined on the factor-space $N=V / M$. The characteristic polynomial of the operator $A$ on the factor-space $N$ is equal to the fraction

$$
P_{\left.A\right|_{N}}(t)=\operatorname{det}\left(1+\left.t A\right|_{N}\right)=\frac{\operatorname{det}\left(1+\left.t A\right|_{V}\right)}{\operatorname{det}\left(1+\left.t A\right|_{M}\right)} .
$$


One can naturally define an action of the operator $A$ on the superspace $V \oplus \Pi M$, where $\Pi$ is parity reversion functor, by setting $A(\Pi \mathbf{v})=\Pi(A \mathbf{v})$. We see that

$$
P_{\left.A\right|_{N}}(t)=\frac{\operatorname{det}\left(1+\left.t A\right|_{V}\right)}{\operatorname{det}\left(1+\left.t A\right|_{M}\right)}=\operatorname{Ber}\left(1+\left.t A\right|_{V \oplus \Pi M}\right)=R_{\left.A\right|_{V \oplus \Pi M}}(t) .
$$

Therefore the rational characteristic function of a linear operator in superspace naturally appears if we consider operators on a factor-space. We have met this phenomenon for the Weingarten operator of hypersurfaces in dual approach (see (15)). One can say that the characteristic polynomial of the Weingarten operator $S$ of a hypersurface in Euclidean space $\mathbb{E}^{n+1}$ can be obtained as the characteristic function of the operator $\mathcal{S}$ extended to $(n+1 \mid 1)$-dimensional superspace.

In the above example the fraction is reducible. The numerator and denominator of the fraction $R_{\left.A\right|_{V \oplus \Pi M}}$ contain a common factor, the polynomial $P_{\left.A\right|_{M}}$.

Proposition 4. Let $A$ be a linear operator on a superspace $V$ and $M$ be an invariant subspace for the operator $A$. Then the characteristic functions of the operator $A$ on the superspaces $V / M$ and $V \oplus \Pi M$ coincide:

$$
R_{\left.A\right|_{V / M}}(t)=R_{\left.A\right|_{V \oplus \Pi M}}(t) .
$$

This simple but important statement demonstrates that a characteristic function can be considered as a multiplicative version of the Euler characteristic. It is this property of Berezinian which makes it an adequate tool for describing Reidemeister torsion. Let us recall its construction. Consider a complex $\left(E=E_{0} \oplus E_{1}, d\right)$ as a superspace. Here the differential $d$ is an odd operator. Denote by $Z$ the kernel of the operator $d$ and by $B$, its image. Then the cohomology of $d$ is $H=Z / B$. Denote by $\operatorname{Ber}(V)$ the space of volume forms on a superspace $V$. Then $\operatorname{Ber}(Z)=\operatorname{Ber}(H) \otimes \operatorname{Ber}(B)$ and $\operatorname{Ber}(E)=\operatorname{Ber}(Z) \otimes \operatorname{Ber}(\Pi B)$, because the differential $d$ is an odd linear operator. Hence the space $\operatorname{Ber}(E)$ is canonically isomorphic to the space $\operatorname{Ber}(H)$. The Reidemeister torsion can be understood as this canonical isomorphism ${ }^{1}$.

We can say something more.

Proposition 5. For an arbitrary (even) operator $A$ on a complex $E=(E, d)$ commuting with the differential d the following equality holds:

$$
R_{\left.A\right|_{H}}(t)=R_{A}(t)
$$

\footnotetext{
${ }^{1}$ This construction was studied by A. S. Schwarz and applied by him in particular to the partition function of a degenerate quadratic functional in Quantum Field Theory (see [4]).
} 
where $H=H(E, d)$ is the cohomology.

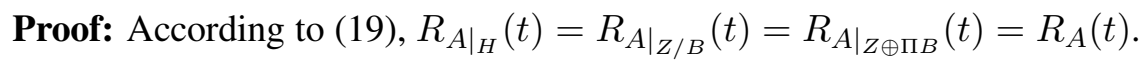

Our considerations reveal that a rational function $R(t)$ such that $R(1)=1$ can be interpreted as the characteristic function of the linear operator on a superspace. Furthermore, if we interpret a linear operator as the Weingarten operator of a surface in a superspace, then this rational function can be considered as a density of supervolume of a tube.

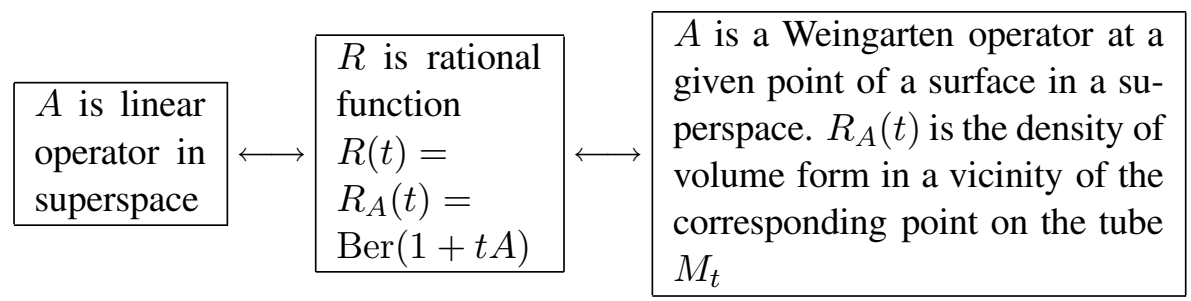

Let us consider an example of a different origin.

Let $X$ be an arithmetic variety given by a polynomial $P_{X}$ in $n$ variables with coefficients in a finite field, say $\mathbb{F}_{p}$ ( $p$ is a prime number). Denote by $\nu_{k}$ the number of points of $X$ over the field extension $\mathbb{F}_{p^{k}} \supset \mathbb{F}_{p}$, i.e., the number of solutions of the equation $P_{X}=0$ in the space $\mathbb{F}_{p^{k}}^{n}$. The zeta-function of an arithmetic variety can be defined as

$$
Z_{X}(t)=\exp \sum_{k=0}^{\infty} \frac{\nu_{k}}{k} t^{k}
$$

(see, e.g., the book [5]). One of the deep results in algebraic number theory is that $Z_{X}(t)$ is a rational function of the argument $t$. It is the first of the famous Weil conjectures, which was proved by Dwork in 1960.

In view of the above we can suggest that this rational function is a characteristic function of a linear operator on a superspace. The properties expressed in Propositions 3 and 4 reveal a cohomological interpretation of this operator. Furthermore, one can interpret this characteristic function as the volume density of a "tubular neighborhood", i.e. an analogue of Weyl tube formula. Philosophically it should not be a surprise, since the definition (20) of the zeta-function can be seen as a formula for the "volume" of a formal neighborhood of a single point. The whole surface $\bar{X} \supset X$ over the algebraic closure of $\overline{\mathbb{F}}_{p} \supset \mathbb{F}_{p}$ can be viewed as a "tubular neighborhood" of this single point. A full understanding of this relation is yet to be achieved. 


\section{Acknowledgements}

I am deeply grateful to Th. Voronov for valuable comments and for encouraging for writing this note. I am also grateful to A. Haunch for interesting discussions.

\section{References}

[1] Candelas P. and de la Ossa X., The Zeta-function of a p-Adic Manifold, Dwork Theory for Physicists, arXiv:hep-th 0705.2056

[2] Gray A., Tubes, Addison-Wesley, USA, 1990

[3] Khudaverdian H. and Voronov T., Berezinians, Exterior Powers and Recurrent Sequences, Lett. Math. Phys. 74 (2005) 201-228.

[4] Schwarz A., Semiclassical approximation in Batalin-Vilkovisky formalism, Commun.Math.Phys. 158 (1993) 373-396

[5] Shafarevich I. Basic Algebraic Geometry. 1. Varieties in Projective Space. 2nd Edition. Springer, Berlin, 1994.

Hovhannes M. Khudaverdian

School of Mathematics

The University of Manchester

Manchester M60 1QD

UNITED KINGDOM

E-mail address: khudian@manchester.ac.uk 